\title{
BMJ Open Experience-based investigation and codesign of approaches to prevent and reduce Mental Health Act use (CO- PACT): a protocol
}

\author{
Roisin Mooney (D) , ${ }^{1}$ Karen Newbigging (D) , ${ }^{1}$ Rose McCabe (D) , ${ }^{2}$ \\ Paul McCrone (D) , ${ }^{3}$ Kristoffer Halvorsrud (D) , ${ }^{4}$ Raghu Raghavan (D) , \\ Doreen Joseph, ${ }^{1}$ Kamaldeep Bhui (i) ${ }^{1}$
}

To cite: Mooney $R$,

Newbigging K, McCabe R, et al. Experience-based investigation and codesign of approaches to prevent and reduce Mental Health Act use (COPACT): a protocol. BMJ Open 2022;12:e060086. doi:10.1136/ bmjopen-2021-060086

- Prepublication history for this paper is available online. To view these files, please visit the journal online (http://dx.doi. org/10.1136/bmjopen-2021060086).

Received 10 December 2021 Accepted 22 December 2021

\section{Check for updates}

(c) Author(s) (or their employer(s)) 2022. Re-use permitted under CC BY-NC. No commercial re-use. See rights and permissions. Published by BMJ.

${ }^{1}$ CHiMES Collborative, Department of Psychiatry, University of Oxford, Oxford, UK ${ }^{2}$ School of Health Sciences, City University of London, London, UK

${ }^{3}$ Institute for Lifecourse Development, University of Greenwich, London, UK ${ }^{4}$ NIHR ARC North Thames, Department of Applied Health Research, University College London, London, UK

${ }^{5}$ School of Nursing and Midwifery, De Montfort University, Leicester, UK

Correspondence to Dr Roisin Mooney; roisin.mooney@psych.ox.ac.uk

\section{ABSTRACT}

Introduction The Independent Review of the Mental Health Act (MHA) in England and Wales confirmed increasing levels of compulsory detentions, especially for racialised communities. This research aims to:

(a) understand the causes of and propose preventive opportunities to reduce the disproportionate use of the MHA, (b) use an adapted form of experience-based codesign (EBCD) to facilitate system-wide changes and (C) foreground the voices of service users at risk of detention to radically reform policy and implement new legislation to ensure the principles of equity are retained.

Methods and analysis This is a qualitative study, using a comparative case study design. This study is composed of five work packages; photovoice workshops will be conducted in eight local systems with service users and healthcare professionals separately (WP1); a series of three EBCD workshops in each local system to develop approaches that reduce detentions and improve the experience of people from racialised communities. This will inform a comparative analysis and national knowledge exchange workshop (WP2); an evaluation led by the patient and public involvement group to better understand what it is like for people to participate in photovoice, codesign and participatory research (WP3); an economic evaluation (WP4) and dissemination strategy (WP5). The impact of the involvement of patients and public will be independently evaluated.

Ethics and dissemination This study is sponsored by the University of Oxford and granted ethical approval from the NHS Research Ethics Committee and Health Research Authority $(21 / \mathrm{SC} / 0204)$. The outputs from this study will be shared through several local and national channels.

\section{INTRODUTION}

People from racialised communities experience more adverse pathways to care, higher rates of compulsory admission and treatment, more contact with the police and criminal justice agencies and poorer long-term outcomes compared with White British people. ${ }^{1-5}$ The precise role of individual-level factors (eg, substance use, comorbidity, cumulative effects of health risk

\section{Strengths and limitations of this study}

- This is a multicentre study that uses participatory methods to foreground the lived experience of people from racialised communities detained under mental health legislation in England.

- The use of creative methods provides unique opportunities to engage community organisations and marginalised populations.

- The comparative case study design enables a detailed understanding of potential approaches and implementation activities need to support improvements.

- The findings will inform the changes to the Mental Health Act and its subsequent implementation to improve the experience and outcomes of people from racialised communities.

- The main limitation is that there is no scope to rigorously evaluate the implementation of changes that may occur as a result of the experience-based codesign. Our proposed approach shows how service users and the public and professionals can unite to provide better experience data; and how creative methods might be used as a policy research tool, especially with marginalised and racialised communities.

behaviours over the life course) and structural inequalities (psychosocial adversity such as school exclusion, victimisation or violence, discrimination and racism) remains uncertain. ${ }^{6}$ There may also be systematic biases adversely affecting people from racialised communities in help seeking, subsequent clinical assessments, perceptions of 'dangerousness' or risk of harm to self $^{578}$ and the appropriateness of mental health support. The forms of support offered may not reflect what matters to service users, and, may, be based on dominant models of illness and care in the global North.

System-based research paradigms can help resolve the contradictions in multiple levels 
of evidence; for example, there is evidence that limited access to effective and high-quality services, stigma and fear of coercion undermine early intervention, ${ }^{9}$ yet conversely the duration of untreated psychosis is shorter in Black ethnic groups. ${ }^{10}$ In exploring the system, it is possible to elicit what works, for whom and in what context to provide a more nuanced understanding of how such contractions may arise and be perpetuated.

A diagnosis of mental illness can have a detrimental effect on future aspirations, owing to social exclusion, the stigma of a diagnosis of mental illness and of being detained. ${ }^{11-13}$ Consequently, poor experiences and outcomes from support, fears about detention and concerns about intervention mean that people may avoid seeking help. Factors influencing the experience of poor mental health converge to act in mutually dependent ways, requiring better research methods that recognise the dynamic interactions of many factors in complex social and health systems; ${ }^{14}{ }^{15}$ for example, a 'syndemic' of anxiety, substance misuse, violence, structural racism, deprivation and psychosis in specific locations may explain a higher rate of psychosis in some ethnic groups ${ }^{8}$ and may explain variations of detentions by place ${ }^{16}$ Efforts to find such causes on national samples producing national policies and practices may be ineffective locally. Furthermore, the specific ethnic groups affected vary by provider and location; hence, an approach grounded in places of high levels of detention is essential to ensure that the findings are interpreted for local actions must reflect local practices, services, demographic profiles, resources and assets. Knowledge from the experiences of service users and other stakeholders is critical to inform preventive approaches that can be tailored to specific populations and places, informing overall national practice and policy recommendations.

\section{Aims}

This study seeks to further research to understand the drivers of rising detention rates and prevention opportunities, taking account of service users' experience and stakeholders' knowledge. These diverse and in-depth experiential perspectives are currently missing from the evidence base.

We will provide an opportunity for people frequently detained under the Mental Health Act (MHA) and professionals using the MHA to share their experiences, using a creative process that permits marginalised perspectives to be shared in a safe and engaging manner. The approach will identify more detailed and nuanced explanations for detention, preventive opportunities and then a codesigned system approach for positive and progressive practice and policy.

\section{METHODS AND ANALYSIS \\ Design}

This is a qualitative comparative case study investigating the experience of people detained under the legal provisions of the MHA, with a particular emphasis on those from racialised communities. We will also gather professionals' experiences of using the MHA. Case study designs and qualitive methods have a rich tradition and enable in-depth and highly valid information about complex systems. ${ }^{17}$ Given the multitude of intersectional influences including demographic, biographical, cultural, clinical, institutional, political, geographical and historical influences on determining pathways to care and ethnic disparities, we propose that this approach is well suited to reveal explanations for inequalities and identify potential preventive approaches.

People who have been detained may find it difficult or impossible to verbalise their feelings, especially if they were traumatised and lost trust in the system of care. In part, the research process itself risks re-enacting power relationships. For example, racialised minorities are often under-represented in research studies. Cocreation and codesign, based on the principle of participatory parity, have the potential to provide a different way of participating in research to generate new knowledge of the complex drivers of inequalities and their interactions. ${ }^{1018} 19$ In our systematic review of participatory method of coproduction and codesign, photovoice emerged as a method with promising evidence of benefit to participants. ${ }^{20}$

Therefore, this research will make use of photovoice (WP1), a community-based participatory research process. We will ask service users and professionals to share experiences and perspectives on causes of detention and preventive opportunities by taking photographs, then reflecting on and captioning these. This creative methodology has been found to be empowering, leaving positive effects on participants' confidence..$^{21}{ }^{22}$ Photovoice offers a safe and supportive process, and the nonverbal creative and aesthetic elements accommodate variations in literacy, language and levels of disability as well as having potential to engage ethnically diverse participants. ${ }^{23}{ }^{24}$ Photovoice has been used with vulnerable populations in mental health settings, in participatory research to change the health systems, and as a method for quality improvement. ${ }^{2025}$ The intention is that photovoice will act as a gentle elicitation process and offer thought provoking images that have the potential to reorientate and motivate the viewer, as opposed to simply and reductively mirroring the dominant narratives that people are accustomed to. ${ }^{26}$ The information from photovoice images and related narratives from service users will feed into experience-based codesign (EBCD) (WP2) ${ }^{27}$ undertaken by local service users, carers, clinicians, police, voluntary organisations, commissioners and policymakers (figure 1). This will consist of a series of three workshops held over 6 months to design an approach to reduce detentions in general, and inequalities by ethnicity. In a fourth national workshop, the participants from each of the case study sites will come together to develop a logic model, programme theory and consider recommendations for the MHA reform. This will include an economic analysis (WP4), consisting of modelling the 


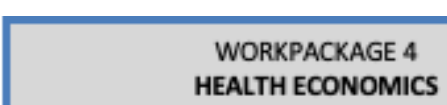

Information about care pathways from photovoice, and information provided during the codesign workshops will enable an evaluation of codesigned outputs will cost to implement

\section{WORKPACKAGE 1 PHOTOVOICE}

In each area 20 service users who have been detained will take part in a series of 3 workshops.

Nationally healthcare professionals will be invited to do the same.

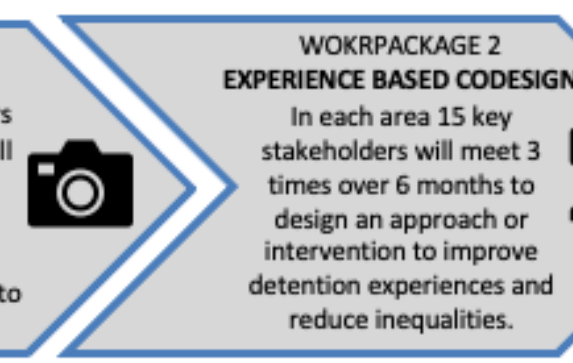

WOKRPACKAGE 2

In each area 15 key

stakeholders will meet 3

times over 6 months to

design an approach or

detention experiences an

reduce inequalities.

WORKPACKAGE 3

PARTICIPANT FEEDBACK

Led by the PPIRG, everyone who takes part in the photovoice and experience based codesign workshops will be asked to provide feedback (in a way that is most accessible to them) about their experiences of participating

This work will be conducted in 8 local systems; London, Lancashire, Leeds, Birmingham, Manchester, Oxford, Derby and Bradford Figure 1 Study design for an experience-based investigation and codesign of approaches to prevent and reduce Mental Health Act use

cost of the new codesigned intervention based on emergent findings, in terms of how it impacts on experiences, service use and associated costs.

We will gain a better understanding of what it is like to take part in participatory action research and EBCD by asking for feedback from everyone who interacts with the study (WP3). The questions asked will be developed by the patient and public involvement (PPI) group.

\section{Setting}

This is a multicentre study and will be conducted in eight local systems: Leeds, Manchester, Birmingham, Oxford, Derby, Bradford, Lancashire and London. The chosen venues for recruitment show variations in the percentage of Black and ethnic minority service users who are detained, and different ethnic group profiles.

\section{Participants}

We will recruit 120-160 service users (15-20 from each local system) with experience of at least one period of care under the MHA in the preceding two years (excluding forensic sections) (table 1). As far as is practically possible, a purposive sample will be constructed in respect of age, ethnicity, gender, type of section and the route into detention that they have experienced. Non-English-speaking people will be encouraged and supported to participate. Community organisations as well as NHS trusts will be engaged to assist with recruitment and ensure that a variety of care pathways are represented.

Twenty professionals who have been involved in using the MHA to detain someone will also participate in photovoice workshops. A broad range of professionals will

Table 1 Photovoice workshops selection criteria

\section{Inclusion criteria}

At least one episode of being detained under the powers of the MHA in the preceding two years, specifically emergency (sections 5 (4), section 5 (2), sections 135 and 136, assessment (section 2) and treatment sections (section 3).

- From one of three broad ethnic groups: White British, South Asian, Black African and Black Caribbean, recognising that there will be diversity within these groups.

- Aged 18 and over.

- Have capacity to provide informed consent.

\section{Exclusion criteria}

- Too distressed to enter a reflective process, currently experiencing a mental health crisis.

- People who have had experience of forensic sections only, as these are likely to be influenced by many other factors related to the nature and type/seriousness of offending and criminal record. However, we will seek to be inclusive and not exclude service users in general adult services, when they have experience of forensic sections. 
Table 2 Experience based co-design workshops selection criteria

\begin{tabular}{ll} 
Inclusion criteria & Exclusion criteria \\
\hline $\begin{array}{l}\text { Involved in providing care to those who are likely to be } \\
\text { sectioned, or have been sectioned under the MHA, within the }\end{array}$ & $\begin{array}{l}\text { No recent (in the last } 12 \text { months) experience of working in } \\
\text { systems surrounding the MHA or being detained. } \\
\text { previous } 12 \text { months. }\end{array}$ \\
From a core discipline involved in care decisions and & commitments preclude sustained involvement. \\
decisions to detain: social work, nursing, psychiatrist, \\
psychologist, doctor, GP, police, advocate, managers of \\
services, and those involved in reviewing MHA detentions \\
such as members of tribunals, administrators for mental \\
health act review.
\end{tabular}

MHA, Mental Health Act.

be represented, including, but not restricted to, police, mental health nurses and psychiatrists.

Once the photovoice workshops have concluded, 120 stakeholders (a minimum of 15 in each local system) will be recruited to participate in local EBCD workshops (table 2). Local partners will collaborate to identify relevant stakeholders. This will include people from across the system, from different perspectives, including service users, carers and professionals. Among the professional participants those who work in the following sectors will be represented, police, voluntary and community, mental health nursing, psychiatry and commissioning.

Participants will be recruited through open adverts and contact with clinicians, clinical research networks, National Institute for Health Research Applied Research Collaboratives, primary care and specialist NHS mental health services. We will recruit through our existing networks in each local system, through charity partners, faith groups, museums, galleries, service user groups, media; and nationally through the Centre for Mental Health, media and service user networks. Service users may bring a carer or friend or confidante with them if they wish, to support them and act as a sounding board or to act as an advocate.

\section{Patient and public involvement}

Patient and public involvement (PPI) is integral to the success of the project, and, therefore, embedded throughout. People with lived experience will contribute to the governance, analysis and dissemination of this project. As such, a Black British woman with lived experience of being detained under the MHA was a coinvestigator on the funding application, is a member of the core project team (who meets on a weekly basis), is employed by the University of Oxford more formally rather than being on a temporary or casual contract, and she coleads the PPI reference group (PPIRG).

PPI should extend beyond service users and carers. Therefore, a voluntary organisation chief executive cochairs the PPI reference group. The PPIRG will meet at key points across the project. There will also be two joint meetings of the advisory board and PPIRG.
Another responsibility of the PPI reference group will be to consider how the experiences of those participating in this project are captured. While there is some evidence that participation in photovoice workshops has many benefits, ${ }^{28}$ this study provides an opportunity to further evidence the impact that taking part in both photovoice and EBCD may have, which can, in turn, inform future research. The research team will work supportively and place authority in the PPI reference group to ensure positive and significant impact on the project. These impacts will be independently evaluated by the Centre for Mental Health.

\section{Data collection}

\section{Photovoice workshops}

In each local system, three photovoice workshops will be held in carefully chosen creative environments that are accessible and facilitate discussion, away from clinical sites. If necessary (owing to government guidance in relation to face-to-face contact as a result of the COVID-19 pandemic), there will be provisions to conduct workshops virtually. Spaces and processes will be reviewed with local teams to ensure that they permit power sharing and full participation.

Participants will be introduced to the concept of photovoice in an introductory workshop, lasting half a day. It will be explained that through images and related narratives, participants will be invited to share their experiences around compulsory admission and treatment. Participants will be provided with disposable cameras, notebooks and prepaid envelopes and asked to post the cameras back to the research team within 2 weeks. Digital options will be facilitated according to participant preference. The notebooks offer a means to write down reflections straight away to aid recall as to why a photo has been taken and what it represents. Offering disposable cameras worked well in our previous work in photovoice. ${ }^{29}$ It did not significantly alter photo quality, while enabling participants to use a simple procedure, and concentrate efforts on the photography. This seemed to incentivise and encourage participation of vulnerable populations (eg, ethnic minority service users), with almost all of the 


\section{Box 1 Photovoice questions}

Describe what you see in this photo.

- How does this photo make you feel?

Why did you take a photo of this?

- What does this photo tell us about your life?

- How can this photo provide opportunities for us and others to improve your life?

- Do you have suggestions for how your care could be improved?

- Within this experience, how is it that you ended up being detained?

- How might detention be prevented?

How can inequalities arise and be reduced?

participants opting to use disposable cameras rather than any digital alternatives.

The second workshop will be held at least 2 weeks after the first, and the third one a week after the second. In any cocreation project, the value of participation may vary for participants at different stages of the process-it may be that the degree of participation that is valuable for a participant may not include full involvement at all stages, and we will pay attention to this in close communication with participants and community representatives. These workshops will last for an entire day, but it will not be mandatory for participants to attend for the entire time. Participants will be presented with their developed images displayed on poster boards or digitally, as they prefer. A guided reflection will be used to assist in generating narrative captions for their images. Prompt questions will be used to elicit their experiences (adapted from Hergenrather, 2009) ${ }^{30}$ (box 1).

This process will take place individually in the second workshop, and then the emerging findings will be shared in larger groups in the third workshop. Discussions in the third workshop will be centred around two questions; 'What made a difference?' and 'What could have been different?'. It will be highlighted that participants are not required to share their experiences and may share as much or as little as they wish to. Previous work has found this process to be very empowering for those participating, owing to the discovery of a different mechanism for sharing their experiences and being understood. In our preliminary work, there was continued engagement, and we have consequently planned for a $23 \%-24 \%$ attrition rate.

Twenty professionals who use the MHA or care for people under the act will be invited to meet on two occasions (an introductory workshop and reflective workshop). These will include psychiatrists, psychologists, social workers, psychologists, nurses, independent mental health advocates, lawyers and police. They will be asked to follow the same photovoice procedure. The content delivered in the introductory workshop will differ slightly as we will be interested in unearthing their experiences of detaining people and practice standards, alongside their perceptions of why ethnic inequalities exist, how to reduce these and prepare services and professionals for this.

\section{EBCD workshops}

The outputs from the photovoice workshops will be analysed and curated to be presented at local EBCD workshops in order to identify significant critical 'touch points'. Three workshops will be convened with a wide range of stakeholders, over a 6-month period, in each local system. The aim of the workshops will be to codesign an approach to reducing detentions (eg, this may include complex, multicomponent and systemic approaches or modifications specifically in practice or one level of the health systems) and consider acceptability, feasibility, barriers to implementation, ethical issues and mechanisms of effect. ${ }^{27}$

The initial codesign workshop will seek to balance the power dynamic between various stakeholders and present knowledge gained from photovoice workshops, to ensure that the service user voice is accurately understood and prevalent throughout the discussion. This workshop will also delineate the process by which codesign principles will be followed, consensus achieved and the rationale for the approach.

The second workshop will be held approximately 1 month after the first. This time frame will enable delegates to process the information presented and consider its relevance while in their day-to-day environment. This second workshop will develop an appropriate theory on which the approach (complex, multicomponent and systemic) will be developed. The participants may suggest specific adaptations to local processes in care, assessment, protections and safeguards and implications for training.

The third workshop will be held approximately 2 to 3 months after the second one. This will consist of all delegates providing feedback from their conversations and experiences of considering the proposed approach in their service settings. This feedback will be discussed and incorporated into the final recommendations, including attention to potential mechanisms, logic models and theory of change, triangulated with the findings of photovoice. Personal experiences of detention, the contexts and mechanisms to improve outcomes will be considered during the refinement of the approach.

A fourth workshop will be convened, including all participants across all the sites. We will bring together the proposed approaches and their experience of barriers and facilitators to implementation, and develop logic models about mechanisms, and theory of change, to codesign an overall approach that will be potentially effective across all sites. To build capacity and facilitate the task of this fourth day of workshops, the research team will provide summaries of evidence of effectiveness drawing from previous research, including a theoretical justification based on systematic reviews, evidence from the independent review of the MHA, for considering proposed approaches. 
Participants in all workshops will be asked whether they consent to being video recorded, and any notes made by participants will be collected after the workshops for analysis. This data will enable the research team to analyse how the principles of codesign were adhered to and inform an analysis of what it is like for people to take part in this type of methodology.

\section{Economic analysis}

Data collected from the photovoice and the first two EBCD workshops will be used for the economic analysis.

First, we will map out care pathways for people who have experienced detention under the MHA. This will be gathered from the narratives shared in WP1. Using information provided, we will identify key events that occur for participants on the way to them being detained. While there are likely to be some similarities between participants (some similarity imposed by the system itself), there will also be unique aspects for each person. By mapping out these pathways, we will be able to see where the events occur that result in costs, whether to the health and social care system or to other agencies and to the participants themselves or their families/friends.

Second, the health economics team will be involved in the EBCD workshops. The key components of such an approach will be identified and the cost implications investigated. The objective will be to estimate the cost per person in receipt of such an intervention or service.

\section{Participant feedback}

All participants will be presented with questions that have been designed by the PPIRG at the end of every workshop (both photovoice and EBCD). We will facilitate different modes for people to provide feedback according to their preference, this could be written, but may also be online or an audio/video recording.

\section{Data analysis}

The data will be in the form of the narratives, images and captions, and reflections of individual participants, a group discussion where service users and carers wish to share their observations, and a video recording (which will also be transcribed) of the reflective workshops of individual or group discussions. Written accounts will also be invited, to produce a rich set of data in a form that service users prefer (eg, post it notes, poetry, text, self-recordings). This will provide rich, detailed data concerning how the information and experiences are both discussed and processed.

Data from the photovoice workshops will be thematically analysed and synthesised to identify patterns and also retaining differences presented in outlying cases, through interim analysis and constant comparison. ${ }^{29} 31$ The aim will be to generate in-depth understandings from the visual and written and recorded materials, generate new theory and qualify existing theory, reveal deeper underlying power structures and symbolic meanings of images, all to identify touch points for codesign.
Thematic analysis will be undertaken on original transcripts coded and held in a qualitative software package. The principles of the 'Framework' approach will be used to extract and organise summary information around the causes of MHA use and preventive opportunities, charts to compare and contrast the findings by different participants: by age, gender, ethnicity, type of section, whether detained or admitted voluntarily, and service user or professional discipline and also the type of image and creative narrative device used. ${ }^{29}$ This will help to map the emergent phenomena across participants and cities and specific narrative and creative methods. The charts are also a convenient way to present summary information for interpretation by participants, the PPIRG and the research team.

A comparative analysis of the outputs from the EBCD workshops will be conducted. Analysis and appraisal of the proposed approaches will be conducted within the workshops alongside the participants. The cost per person in receipt of new approaches will be estimated, and the impact on the pathway will be modelled by a health economist. The potential impact of the approach will be discussed within the workshops. The outputs will consist of co-designed locally implementable interventions that aim to reduce inequalities in the implementation of the MHA.

Feedback gathered from everyone who interacts with the study will be thematically analysed by the PPI lead who is an expert by experience alongside the other coapplicants on the research programme.

\section{Ethics and dissemination}

The innovation to be emphasised in this research is the use of photovoice as a method of identifying touch points in relation to detention under the MHA that will be explored in further depth in EBCD workshops. Internal experiences and feelings that are difficult to verbalise are given voice through photography; the creative process enables emotional activation and safety when talking about potentially stigmatising issues where language sometimes fails. These materials then feed into EBCD to identify what needs to change and how.

It is potentially more challenging to conduct photovoice in mental health settings due to ethical issues, notably participants' desire for anonymity; the complexity of services; and challenges around improving experiences in complex systems. ${ }^{27}$ Thus, by using photovoice as a starting point, service users will have control over the experiences that they share and the opportunity to share these in a protected space that we will provide. The depth of narratives will evolve with sufficient time, and presented in written, audio or verbal form that participants feel comfortable with.

Those who do not have the capacity to consent for themselves will be excluded through careful consideration within an experienced team and in close consultation with community representatives. This judgement will be made in part by the recruiting clinical or NGO 
with which service users are engaged, but if necessary, at the stage of providing information and securing consent. Those receiving consent will be sensitive to these judgements being dynamic and needing review.

The research team will work with local care teams and community organisations to ensure that the recruitment strategy is inclusive and facilitates participation from marginalised populations. Potential participants will be presented with an information sheet detailing the exact nature of the study; what it will involve for the participant; any risks involved in taking part. It will be clearly stated that the participant is free to withdraw from the study at any time for any reason without prejudice to future care, and with no obligation to give the reason for withdrawal. Fully informed consent will be received at the beginning of the first photovoice and EBCD workshops. Willingness to continue with the study will be confirmed with participants at subsequent workshops. In all workshops, participants will be reminded that their contributions will be confidential and made anonymous if shared beyond workshops, unless they explicitly ask to waive their anonymity. Photovoice has been found to be an empowering process, and it is important to ensure that once the workshops have concluded that participants are not then disempowered should they wish to remain associated with their photographs.

Knowledge sharing and collaborative working will be prominent for the duration of the project. It will be important to ensure that the principles adopted are not diluted and that any press or social media involvement frames the messages generated in a way that does not disempower or further marginalise our participants. ${ }^{32}$ The outputs from this work will be shared through a number of both local and national channels. There is a dedicated study website and Twitter account (@co_pact), alongside a monthly newsletter that will ensure continued awareness from a wide variety of stakeholders. Exhibitions of the photographs will be held in local systems as well as presented at seminars to raise awareness of the study and the issues surrounding severe mental illness and ethnic inequalities. Alongside traditional publications in academic journals to advance and stimulate discussion surrounding this innovative use of creative methodology, articles will be written in collaboration with community organisations in each of the local systems.

Information from the proposed research will inform implementation of the recommendations from the Independent Review of the MHA.

Twitter Roisin Mooney @Roisin_mm_, Karen Newbigging @NewbiggingKaren, Rose McCabe @RoseMcCabe2, Paul McCrone @mccrone_paul, Raghu Raghavan @RaghuRaghavan1 and Kamaldeep Bhui @KSBhui

Contributors RM drafted the initial manuscript. RM, KB and KH conceptualised the project. $\mathrm{KN}$ and $\mathrm{RMcC}$ contributed to early versions of the manuscript. PMcC, RR, $\mathrm{KN}, \mathrm{KB}$ and $\mathrm{RMCC}$ all reviewed the final manuscript. DJ contributed towards the PPI strategy and reviewed the manuscript. All authors were involved in developing the successful funding application to conduct this research and are currently leading different work packages.
Funding This study/project is funded by the National Institute for Health Research (NIHR) Policy Research Programme (NIHR201704).The views expressed are those of the authors and not necessarily those of the NIHR or the Department of Health and Social Care

\section{Competing interests None declared.}

Patient and public involvement Patients and/or the public were involved in the design, or conduct, or reporting, or dissemination plans of this research. Refer to the Methods section for further details.

Patient consent for publication Not applicable.

Provenance and peer review Not commissioned; externally peer reviewed.

Open access This is an open access article distributed in accordance with the Creative Commons Attribution Non Commercial (CC BY-NC 4.0) license, which permits others to distribute, remix, adapt, build upon this work non-commercially, and license their derivative works on different terms, provided the original work is properly cited, appropriate credit is given, any changes made indicated, and the use is non-commercial. See: http://creativecommons.org/licenses/by-nc/4.0/.

Author note Study Website: https://www.psych.ox.ac.uk/research/chimes/co-pact; Twitter handle: @co_pact and e-mail address co-pact@psych.ox.ac.uk

\section{ORCID iDs}

Roisin Mooney http://orcid.org/0000-0001-5792-3314

Karen Newbigging http://orcid.org/0000-0001-8764-528X

Rose McCabe http://orcid.org/0000-0003-2041-7383

Paul McCrone http://orcid.org/0000-0001-7001-4502

Kristoffer Halvorsrud http://orcid.org/0000-0002-8813-0939

Raghu Raghavan http://orcid.org/0000-0002-3346-0877

Kamaldeep Bhui http://orcid.org/0000-0002-9205-2144

\section{REFERENCES}

1 Barnett $\mathrm{P}$, Mackay E, Matthews $\mathrm{H}$, et al. Ethnic variations in compulsory detention under the mental health act: a systematic review and meta-analysis of international data. Lancet Psychiatry 2019;6:305-17.

2 Weich S, McBride O, Twigg L, et al. Variation in compulsory psychiatric inpatient admission in England: a cross-classified, multilevel analysis. Lancet Psychiatry 2017;4:619-26.

3 Bhui K, Halvorsrud K, Nazroo J. Making a difference: ethnic inequality and severe mental illness. Br J Psychiatry 2018;213:574-8.

4 Halvorsrud K, Nazroo J, Otis M, et al. Ethnic inequalities and pathways to care in psychosis in England: a systematic review and meta-analysis. BMC Med 2018;16:223.

5 Morgan C, Knowles G, Hutchinson G. Migration, ethnicity and psychoses: evidence, models and future directions. World Psychiatry 2019;18:247-58.

6 Nazroo JY, Bhui KS, Rhodes J. Where next for understanding race/ ethnic inequalities in severe mental illness? Structural, interpersonal and institutional racism. Sociol Health IIIn 2020;42:262-76.

7 Bhui K, Ullrich S, Kallis C, et al. Criminal justice pathways to psychiatric care for psychosis. Br J Psychiatry 2015;207:523-9.

8 Coid J, Gonzalez Rodriguez R, Kallis C, et al. Ethnic disparities in psychotic experiences explained by area-level syndemic effects. $\mathrm{Br} J$ Psychiatry 2020;217:555-61.

9 Parkman S, Davies S, Leese M, et al. Ethnic differences in satisfaction with mental health services among representative people with psychosis in South London: prism study 4. Br J Psychiatry 1997;171:260-4.

10 Bhui K, Ullrich S, Coid JW. Which pathways to psychiatric care lead to earlier treatment and a shorter duration of first-episode psychosis? BMC Psychiatry 2014;14:72.

11 Sayce L. Stigma, discrimination and social exclusion: what's in a word? J Ment Health 1998;7:331-43.

12 Clement S, Schauman O, Graham T, et al. What is the impact of mental health-related stigma on help-seeking? A systematic review of quantitative and qualitative studies. Psychol Med 2015;45:11-27.

13 Tsang HWH, Ching SC, Tang KH, et al. Therapeutic intervention for internalized stigma of severe mental illness: a systematic review and meta-analysis. Schizophr Res 2016;173:45-53.

14 Diez Roux AV. Complex systems thinking and current impasses in health disparities research. Am J Public Health 2011;101:1627-34.

15 Petticrew M, Rehfuess E, Noyes J, et al. Synthesizing evidence on complex interventions: how meta-analytical, qualitative, and mixed-method approaches can contribute. J Clin Epidemiol 2013;66:1230-43. 
16 Bhui K, Halvorsrud K, Mooney R, et al. Is psychosis a syndemic manifestation of historical and contemporary adversity? Findings from UK Biobank. Br J Psychiatry 2021;219:686-94.

17 Bahari SF. Qualitative versus quantitative research strategies: contrasting epistemological and ontological assumptions. Sains Humanika 2010;52.

18 Mendenhall E. Beyond comorbidity: a critical perspective of Syndemic depression and diabetes in cross-cultural contexts. Med Anthropol Q 2016;30:462-78.

19 Tsai AC, Mendenhall E, Trostle JA, et al. Co-Occurring epidemics, syndemics, and population health. Lancet 2017;389:978-82.

20 Halvorsrud K, Kucharska J, Adlington K, et al. Identifying evidence of effectiveness in the co-creation of research: a systematic review and meta-analysis of the International healthcare literature. J Public Health 2021;43:197-208.

21 Han CS, Oliffe JL. Photovoice in mental illness research: a review and recommendations. Health 2016;20:110-26.

22 Halvorsrud K, Rhodes J, Webster GM, et al. Photovoice as a promising public engagement approach: capturing and communicating ethnic minority people's lived experiences of severe mental illness and its treatment. BMJ Open Qual 2019;8:e000665.

23 Shumba TW, Moodley I. Part 1: a review of using photovoice as a disability research method: implications for eliciting the experiences of persons with disabilities on the community based rehabilitation programme in Namibia. Afr J Disabil 2018;7:418.
24 Budig K, Diez J, Conde P, et al. Photovoice and empowerment: evaluating the transformative potential of a participatory action research project. BMC Public Health 2018;18:432.

25 Overmars-Marx T, Thomése F, Moonen X. Photovoice in research involving people with intellectual disabilities: a guided photovoice approach as an alternative. J Appl Res Intellect Disabil 2018;31:e92-104.

26 Coffey J. Embodied methodologies: photo-voice, affect, and the body. In: Coffey J, ed. Everyday embodiment: rethinking youth body image. Cham: Springer International Publishing, 2021: 25-45.

27 Larkin M, Boden ZVR, Newton E. On the brink of genuinely collaborative care: experience-based Co-Design in mental health Qual Health Res 2015;25:1463-76.

28 Barry J, Monahan C, Ferguson S, et al. "I came, I saw, I conquered": reflections on participating in a PhotoVoice project. J Ment Health Train Educ Pract 2021;16:257-68.

29 Pope C, Ziebland S, Mays N. Analysing qualitative data. BMJ 2000;320:114.

30 Hergenrather KC, Rhodes SD, Cowan CA, et al. Photovoice as community-based participatory research: a qualitative review. Am J Health Behav 2009;33:686-98.

31 Sundler AJ, Lindberg E, Nilsson C, et al. Qualitative thematic analysis based on descriptive phenomenology. Nurs Open 2019;6:733-9.

32 Groot BC, Schrijver J, Abma TA. Are you afraid of press and social media? Ethics in photovoice in participatory health research. Educ Action Res 2021;23:1-19. 